\title{
APROXIMACIÓN TEÓRICA PARA ENTENDER LA VIOLENCIA DESDE UN ENFOQUE CRÍTICO
}

\author{
Martha Elisa Nateras González* \\ https://orcid.org/0000-0002-7045-1400
}

RECIBIDO: Septiembre 2020 / ACEPTADO: Febrero 2021 / PUBLICADO: Mayo 2021

Como citar: Nateras González, Martha (2021). Aproximación teórica para entender la violencia desde un enfoque crítico. Telos: revista de Estudios Interdisciplinarios en Ciencias Sociales, 23 (2), Venezuela. (Pp.305-324).

DOI: www.doi.org/10.36390/telos232.07

\section{RESUMEN}

Encontrar una definición ampliamente reconocida o legitimada de la violencia es poco probable por varias razones, entre ellas, porque es un concepto multifacético y multidimensional y porque la ampliación o reducción de esta locución zanja su valor descriptivo y explicativo, debilitando su impacto. No obstante, es fundamental entender el sentido; el ejercicio, y los detonantes de la violencia, y el punto de partida es tratar de acercarse a un concepto lo suficientemente capaz de yuxtaponer su mejor comprensión. En tal sentido, el objetivo de este artículo es llevar a cabo un acercamiento teórico de la violencia, a partir de la exploración de las aportaciones teóricas de algunos autores que analizan o retoman este concepto desde una perspectiva crítica, centrando la atención en el análisis que hace de la violencia Slavoj Žižek $(2008,2009)$, se retoma como autor central debido a que sus textos son imprescindibles en el pensamiento crítico. La principal conclusión de este texto es que, sin subestimar la perspectiva teórica con que se examine la violencia -ya sea como el resultado de las crisis de un sistema, la consecuencia del cálculo estratégico de un actor racional o como producto de la estructura socio-cultural- para tener los elementos necesarios y lograr un mejor conocimiento del fenómeno de la violencia, es necesario ligarla a otros conceptos como: poder, conflicto, dominación, explotación y discurso hegemónico, los cuales se destacan a lo largo de este documento.

Palabras clave: violencia, conflicto, violencia simbólica, violencia estructural.

\footnotetext{
* Facultad de Ciencias Políticas y Sociales, Universidad Autónoma del Estado de México. Correo electrónico: marnateras@yahoo.com.mx
} 


\title{
Theoretical approach to understand violence from a critical approach
}

\begin{abstract}
Finding a widely recognized or legitimized definition of violence is unlikely for several reasons, including because it is a multifaceted and multidimensional concept and because broadening or reducing this phrase ditch its descriptive and explanatory value, weakening its impact. However, it is essential to understand the meaning; the exercise, and the triggers for violence, and the starting point is to try to get close to a concept capable enough to juxtapose its best understanding. In this sense, the objective of this article is to carry out a theoreticalmethodological approach to violence, based on the exploration of the theoretical contributions of some authors who analyze or take up this concept from a critical perspective, focusing on the Slavoj Žižek's analysis of violence $(2008,2009)$ is retaken as the central author due to the fact that his texts are essential in the critical thinking. The main conclusion of this text is that, without underestimating the theoretical perspective with which violence is examined - either as the result of the crises of a system, the consequence of the strategic calculation of a rational actor or as a product of the social-cultural structure - to have the necessary elements and achieve a better understanding of the phenomenon of violence, it is necessary to link it to other concepts such as: power, conflict, domination, exploitation and hegemonic discourse, which are highlighted throughout this document.
\end{abstract}

Keywords: violence, conflict, symbolic violence, structural violence.

La violencia física siempre deja huellas visibles; la violencia simbólica no necesariamente deja estas huellas.

\section{Introducción}

(Wieviorka, 2001, p.338)

La violencia es un elemento inherente a la historia de la humanidad (Pessin, 1979) al ser propia del ser humano (Domenach, 1981), desde un enfoque reduccionista, la violencia es vista únicamente como agresividad (Klineberg, 1981) que obedece solo a la voluntad de un actor, despojándola de esta manera del atributo involuntario que pueda tener, tornándola una acción deliberada en perjuicio de alguien. La violencia es un concepto complicado de definir, debido a que es multifacético; porque en algunas ocasiones se refiere a la violencia en singular o a las violencias en plural (Isla y Míguez, 2003); por la fragilidad entre los límites entre lo que es violencia y lo que no lo es, y por la importancia del contexto desde donde se observan las violencias.

Hoy en día se ha vuelto común la adjetivación del acto para fines analíticos, así como para marcar distancias entre las distintas formas en que se ejerce la violencia; es así que escuchamos hablar de violencias: política, social, cultural, económica, delictiva, laboral, escolar, familiar, de género ${ }^{1}$, racial o étnica, religiosa, policial, institucional, entre otras (Garriga

\footnotetext{
${ }^{1}$ El tema de la violencia por razones de género tiene en la actualidad un lugar preponderante, entre otras cosas, porque esta reúne varias formas de violencia debido a que está arraigada en el conjunto de costumbres y prácticas machistas y misóginas, que imponen cánones de una masculinidad basada en el uso del poder y la fuerza que producen diversas formas de violencia, desde la física, la
} 
y Noel, 2010).

El punto es que el incremento en los niveles de violencia han generado una emergencia, sobre todo para la Ciencias Sociales, para tratar de analizarla y por ende para proponer alternativas de solución. El problema del acercamiento a este tema es que, inevitablemente, en el trabajo de investigación siempre se termina uno topando con la complejidad del fenómeno, no sólo por los datos que dan cuenta de la problemática, más aún, con el aparato teórico-metodológico para abordarla y permita ir más allá de las cifras. Lo cierto es que la incidencia delictiva se ha vuelto un indicador de los niveles de violencia que tiene un país y a pesar que las cifras son necesarias no siempre muestran la gravedad de este fenómeno social y por consiguiente de su tratamiento. La criminalidad hay que describirla y comprenderla, debido a que explica parte de esta violencia, pero si la atención se centra exclusivamente en los actos de violencia directa se corre el riesgo de no entenderla en su exacta dimensión, así como sus implicaciones.

De acuerdo con Boaventura De Sousa (2003), todos los conceptos con que representamos la realidad y a través de los cuales constituimos las diferentes ciencias sociales y sus conceptos, entre ellos el de violencia, tienen condicionantes espacial, física y simbólica, al tener claro esto nuestros instrumentos analíticos permitirán una mejor comprensión de las relaciones sociales en las que se elaboran cada uno de los conceptos. De esta forma, el imaginario de lo real espacial puede convertirse en la matriz para comprender todos los aspectos de la realidad.

La primera dificultad para la comprensión y análisis de la violencia es la de contar con una acepción lo suficientemente capaz de acercarnos a su mejor discernimiento. Sobre todo si se considera que el uso de la fuerza física suele ser considerado como el principal detonador de las situaciones de violencia, por ello esta visión del problema es la que empieza a complejizar su significado, por ende esta perspectiva restringida de ciertos hechos sociales es la que justifica que se analice a través de un enfoque crítico. Considerando que no hay una teoría o un enfoque específico para su tratamiento, es fundamental considerar que la violencia es resultado de factores etológicos (es decir, producto de la conducta, el instinto o de las relaciones entre los seres humanos); psicológicos; psicosociales; simbólico-culturales; políticos; históricos; semiológicos, y éticos, como punto de partida (Aróstegui, 1994).

Encontrar una definición aceptada y aprobada es poco probable, por ser un concepto multifacético y multidimensional, como ya se dijo; no obstante, el "aumento indiscriminado de la ampliación del término acaba por debilitar su valor descriptivo y explicativo" (Aróstegui, 1994, p. 22) (Platt, 1992). Desde algunas disciplinas como la Antropología, Sociología, la Ciencia Política, la Psicología o la Historia, se han hecho esfuerzos importantes, en términos conceptuales, para entender qué es la violencia.

Coady (1986), distingue tres tipos de definiciones de la violencia: definiciones restringidas u observacionales; legitimistas 0 estrictas, y amplias 0 expansivas. Las primeras son las más comunes, ya que hacen referencia o se relacionan con el uso de la fuerza física directa, identificándola con actos de violencia interpersonal, como un acto intencional para

emocional, sexual y económica, entre otras, que pueden derivar en el feminicidio (Incháustegui y López, 2012). El femicidio al ser una herramienta del patriarcado, también contribuye a instrumentalizar el racismo, la opresión económica, la xenofobia, la heteronormatividad e incluso el colonialismo (Sagot, 2013). 
provocar daño físico (Osorio, 2012)2. Las posiciones legitimistas o estrictas surgen con el pensamiento político conservador, entienden la violencia como el uso de la fuerza de manera ilegal e ilegitima y su aceptación como coacción de Estado. Las definiciones amplias o expansivas consideran a la violencia como parte de la estructura socio-cultural, por tanto están ligadas a las nociones estructurales de violencia, en donde ésta es equiparable a toda situación de dominación. Para este tipo de definiciones la violencia es la existencia de conflicto, en consecuencia el conflicto tiene una naturaleza violenta, por eso no hacen ninguna distinción entre ambos conceptos, la diferencia entre ambas se abordará en el siguiente apartado.

Un punto débil para entender la violencia desde la ciencia política -porque según Moore (1994), está subteorizada- es que su terminología no distingue entre palabras fundamentales para su comprensión, tales como: poder, potencia, fortaleza, fuerza, autoridad e incluso violencia se emplean como sinónimo porque tienen la misma función (Arendt, 2006). La cuestión es que cada una hace alusión a distintas situaciones y el término a que se refiere cada situación no existiría sin la multiplicidad de fenómenos sociales. Un ejemplo de esto son cómo las teorías de la violencia civil, desarrolladas por los clásicos, para entender la violencia, han acudido a los conceptos sociales, a las teorías sobre el sistema social, a las teorías políticas, a las teorías de comportamiento psicológico o a las que hablan sobre las formas de la cultura. En esa interacción entre disciplinas es posible reconocer la violencia civil como el resultado de numerosos procesos sociales y al mismo tiempo permite diferenciar los aportes teóricos de cada una para identificar si tienen cierto valor predictivo y saber cuándo es posible un brote de estos, ante cierto contexto y circunstancias específicas (Aróstegui, 1994).

Arendt (2006), señala que para todos los teóricos políticos, tanto de izquierda como de derecha, la violencia es la más incuestionable manifestación de poder, pero esto no significa que la violencia y el poder sean iguales. La violencia tiene un carácter instrumental y fenomenológicamente se acerca a la potencia, por consiguiente sus instrumentos son utilizados para multiplicar esa potencia.

Un ejemplo, es la violencia civil como una noción que hace referencia a la violencia en la sociedad, como parte de las relaciones sociales y de la correlación de fuerzas en el sistema social. El carácter civil excluye situaciones de violencia planificada de forma estratégica, es decir, de guerra normalizada y de enfrentamientos entre sociedades distintas, pues más bien es una cuestión interna (Aróstegui, 1994).

Hoy tal parece que la realidad política tiene su soporte en la violencia organizada instalada en un espacio geográfico, fuera del orden simbólico de naturaleza política. La violencia física concentrada fuera de la escena, parece confirmarse como la razón última del sentido de la realidad política (Ávalos, 2020). Debido a que la violencia física se define a través del uso material de la fuerza excesiva, voluntariamente perpetrada en menoscabo de alguien en la consecución de un fin (Chenais, 1981; Fisas, 1987), esta forma es la que adquiere el poder para Weber (1991), como la capacidad de influir en las decisiones de otros actores

\footnotetext{
2 El uso de la fuerza física con la intención de causar daño es la definición más cercana o visible, en este sentido para Garretón la violencia es "la manifestación o ejercicio inadecuado de la fuerza o poder, ya sea por la extemporaneidad o por la desmesura, no existiendo intencionalidad por parte del agente o del paciente de la acción" (en Pérez, 2017, p. 241).
} 
sociales en beneficio de quien detenta el poder (Castells, 2009), y por lo tanto el ejercicio de la violencia.

Por otro lado, historiográficamente es posible identificar la existencia de períodos en donde la presencia de situaciones violentas sea más visible o también el cambio en las estructuras ideológicas e instrumentales de la violencia, pero es necesario de otras disciplinas para poder identificar los distintos escenarios en que se presenta la violencia, lo cual sin duda es relevante para un mejor análisis (Aróstegui, 1994).

Considerando lo ya expuesto, el objetivo de este artículo es hacer una revisión teórica de la violencia, a partir de la exploración de algunos teóricos que analizan o retoman dicho concepto desde una perspectiva crítica, centrando la atención en el análisis que hace de la violencia Slavoj Žižek $(2008,2009)$. El autor central de esta aproximación teórica es este filósofo esloveno debido a que sus textos son imprescindibles en el pensamiento crítico y porque su enfoque no se ciñe "al fácil recurso de aceptar al capitalismo y proponer reformas que lo hagan más humano: la inclusión del otro, los derechos humanos, el multiculturalismo, la sociedad civil, etcétera, son analizados y criticados acremente por él" (Ávalos, 2020, p. 380).

Se retoma la perspectiva crítica, porque como bien lo refiere Boaventura De Sousa (2003), las ciencias sociales hoy enfrentan una gran dificultad, vinculada con la realidad que hoy vivimos en todo el mundo y su problemática, la cual es imprescindible criticar. Esa realidad, cualquiera que sea la forma en que se conciba, es necesario que se analice a través de la teoría crítica, debido a que no reduce la "realidad" a lo que existe y porque su tarea principal es "definir y valorar la naturaleza y el ámbito de las alternativas a lo que está empíricamente dado" (De Sousa, 2003, p. 23).

Para cumplir con el objetivo planteado, primero se establece una correlación entre conflicto y violencia para señalar la diferencia entre ambos conceptos, para ello se retoma el pensamiento de algunos autores contemporáneos que analizan el conflicto desde la teoría social, debido a los elementos que aportan para establecer esta distinción. Posteriormente se aborda a la violencia como objeto de estudio desde las distintas disciplinas sociales que analizan el comportamiento y la interacción social como: la sociología, la antropología, la psicología y la teoría política; estas disciplinas se exploran con la finalidad de acercar el análisis a las violencias que distingue con claridad Žižek (2009): sistémica, simbólica y subjetiva. En el último punto se retoma el análisis que hace de la violencia Žižek (2009), quien ha forjado una nueva perspectiva al pensamiento crítico por su modo de interpretar a diversos pensadores, así como por el abordaje que hace de diferentes temas y sucesos, esto lo hace un referente indiscutible para entender el mundo actual, en donde los datos y la información precisan de una guía que nos haga pensar (Ávalos, 2020), es decir, su enfoque teórico provee de los elementos necesarios para inspeccionar la estructura socio-cultural que condiciona 0 determina la violencia.

\section{Conflicto y violencia: el uso indistinto de ambos conceptos}

El uso análogo de ambos conceptos puede provocar más que confusiones, su utilización como sinónimos puede derivar en un análisis errado de la violencia. Desde un enfoque histórico y social el conflicto es un componente inherente del proceso social, ya algunos autores contemporáneos de la teoría del conflicto, como Dahrendorf, Rex, y Coser, han caracterizado el conflicto, sus funciones, la dinámica en que se desenvuelve, los principios 
básico para solucionarlo y el uso de la violencia (Aróstegui, 1994). La violencia en esta lógica se produce en el seno de un conflicto, sin que ésta sea necesariamente el resultado del último; por tanto, conflicto y violencia no son equivalentes, no obstante es común la asociación o el uso indistinto de ambos conceptos. El problema más allá de la confusión entre términos, es que erróneamente se piensa que la violencia fragmenta o divide los intereses de los individuos, sin embargo, esta función la ha llevado a cabo previamente el conflicto, la violencia surge cuando no se tiene otros instrumentos para resolver el conflicto.

Dahrendorf (1962), ubica el conflicto principalmente en el campo político-social, pues para él, la causa que desata un conflicto entre el colectivo social son las relaciones de poder y de dominio, debido a que son asimétricas y esto dará pie a la conformación de dos facciones: por un lado los que buscan permanecer en el poder y por medios legítimos mantener su dominio y los que se resisten a ello, este es el motor de la historia. Este autor reconoce que la formación de estos dos bandos no es mecánica, sino que su integración obedece a dos cuestiones: a los intereses latentes (conciencia para si) y a los intereses manifiestos (conciencia en si) del conjunto social. Los intereses latentes son el resultado de la relación asimétrica de poder, en cambio los intereses manifiestos expresan las aspiraciones especificas del colectivo; en su propuesta teórica los intereses manifiestos impulsan a un conflicto probable, sin que esto derive en un acto violento necesariamente.

Para Coser (1961), no se puede concebir el devenir social sin el conflicto, por tanto centra su atención en las funciones del conflicto y no tanto en el origen del mismo. No obstante, establece que el conflicto es el resultado de la distribución diferencial de derechos y privilegios, el cual se detona cuando los que carecen de esos privilegios no acepten esa distribución diferenciada. Entre una de las funciones fundamentales del conflicto está la que permite la integración de grupos y coadyuva en la cohesión interna, a partir de ésta se puede distinguir entre "nosotros" y los "otros", dando lugar a la ideología. Estos mecanismos de identidad e integración interna son fundamentales en la formación del conflicto, pues para Coser a mayor lealtad o intimidad en un grupo hay una tendencia proporcional a reprimir la hostilidad para conservar la unión; lo cual no implica que la hostilidad genere un conflicto en automático, ya que para que esto pase es necesario la interacción entre parte hostil y el objeto antagónico. Para evitar ese antagonismo son fundamentales las instituciones, pues tienen la función de ser "válvulas de seguridad" para canalizar la hostilidad, entre menos "flexible" y más "intolerante" es la sociedad se requiere de más instituciones para regularla.

Asimismo, para Coser los conflictos pueden ser un fin o un medio, no obstante, casi siempre se da una fusión de ambos. El conflicto da lugar a asociaciones y coaliciones, permanentes o fugaces, dependiendo de la naturaleza del conflicto, generalmente estas asociación que surge del conflicto sólo tiene por objetivo aumentar la supervivencia de los grupos. No obstante, la estructura y finalidades del grupo definen su capacidad de enfrentar conflictos internos y externos; el establecimiento de conflictos liga a los contendientes, estableciendo relaciones donde antes no existían.

Para Rex (1985), el conflicto es una acción intencional para mover la voluntad de algún actor específico, en donde está presente la resistencia, el detonante del conflicto para este autor es la escasez y el poder. Distingue tres etapas en la relación conflictiva: el consenso, la utilización de la fuerza y la legitimidad. Una aportación fundamental de Rex en la dinámica del conflicto es la negociación y los argumentos "racionales" en este proceso, los 
cuales están permeados por los sentimientos. Sin embargo, reconoce que la imposibilidad de llegar a un arreglo en el proceso conflictivo tiene que ver con las características culturales de los sujetos intervinientes.

Lo importante es dejar claro que el conflicto precede a violencia, pero no como una relación automática de causa-efecto, porque un conflicto puede existir sin que jamás se manifiesten situaciones de violencia. Por tanto, la violencia no es un resultado inevitable del conflicto, Álvarez (2013) señala que, considerando que no todos los conflictos derivan en violencia, es fundamental observar a la violencia como una forma de resolver conflictos y como edificadora de lazos sociales, no solo como una fuerza perturbadora que genera cambios bruscos (Harris, 1994; Harvey, 1994; Álvarez, 2013).

Según la teoría,

puede haber conflictos sin violencia, conflictos que no alcancen la situación de violencia, conflictos resueltos sin violencia, en el sentido de la inexistencia de una imposición, o, en último caso, del uso de fuerza física, de fuerza explícita, como caracterizadoras de la violencia. Y puede haber en otros casos, por el contrario, conflictos de tan difícil resolución que den lugar a un amplio y duradero empleo de la violencia. La violencia, en fin, tiene, sin duda, como manifestación esencial el uso de esa fuerza física, pero tampoco ello es su característica constitutiva (Aróstegui, 1994, p. 30).

En este sentido, de acuerdo con Wieviorka (2001), la violencia es lo contrario de una relación conflictiva, es en realidad el no conflicto, debido a que entre más se conflictualice una demanda social, menor cabida tendrá la violencia. De hecho, según este autor, la violencia precedió a la integración del movimiento sindical, al respecto señala que "históricamente el movimiento obrero ha sido menos violento cuando ha sido capaz de conflictualizar su relación con el enemigo de clase" (Wieviorka, 2001, p. 343). A diferencia de Wieviorka, Sorel (2005), considera que la naturaleza de la clase obrera debe ser violenta, en tanto actor contestatario, para que no pierda su dinamismo y por ser el medio a través del cual lograrán su triunfo. No obstante, también la burguesía debe ser violenta, pues con esa cualidad de ambas partes es que se logra más civilización, por tanto para Sorel ${ }^{3}$ el conflicto en sí es violencia, perspectiva que no comparte Wieviorka. Por otra parte, para Frantz Fanon (2007), la violencia es el medio para salir de la relación conflictual, producto de la dominación, pues en esta relación social no tiene cabida la negociación.

Desde la perspectiva legitimista, el uso de la fuerza no es sinónimo de uso de la violencia por parte del poder político, pues éste está cubierto de halo de la legitimidad; es decir, el uso de la fuerza es un medio para la resolución de los conflictos sociales y la violencia se emplea ante la necesidad de establecer o restablecer el orden que se perdió por la presencia del conflicto. El uso de la fuerza por parte del poder político, en la resolución del conflicto, no es necesario que se consensué, pues está dirigido a mantener el status quo, en tanto que el uso de la violencia por parte de agentes no estatales que cuestionan ese orden establecido es sublevación (Aróstegui, 1994). Desde esta lógica, el uso de la violencia por parte de las instituciones del Estado, no solo es legítimo 4 es un medio para combatir y eliminar el conflicto

\footnotetext{
${ }^{3}$ Sorel "cree firmemente que la violencia subyace como principio fundamental de la propia sociedad moderna, en tanto que sus instituciones han sido fundadas por actos de violencia" (Arteaga y Arzuaga, 2017, p. 24).

${ }^{4}$ Para Riches (1988) la legitimidad de la violencia genera desacuerdos, debido a que lo que para algunos es legítimo para otros no lo
} 
social.

En los términos de Hook la coerción física empleada por la «autoridad debidamente constituida» es legítima y debería ser llamada «fuerza», mientras que el término violencia debería ser aplicado solo a los actos de coerción física ilegal...En la posición de Walter...la violencia será «prescrita socialmente y definida como legítima cuando se trata de control o castigo, de acuerdo con prácticas que nos son familiares y de forma que el daño destructivo es medido y sus límites expuestos claramente» (Aróstegui, 1994, p. 28).

Lo importante de la relación conflicto y violencia es que, en las distintas propuestas teóricas revisadas, el punto de partida, para explicar la violencia, es la existencia del conflicto. Sin embargo, considerando que el concepto a analizar en este texto es el de violencia y que el objetivo es hacer una revisión teórica, se llevará a cabo una exploración de las disciplinas sociales que analizan la violencia.

\section{La violencia como objeto de estudio desde la óptica de distintas disciplinas sociales}

Como ya se comentó, la realidad que hoy vivimos a nivel mundial y su problemática, entre ella el incremento en los niveles de violencia, han inducido a las ciencias sociales a profundizar en el tema para intentar proponer alternativas de solución, para lo cual, desde la perspectiva de quien escribe, es imprescindible hacerlo desde una postura crítica.

Las teorías sociológicas han vertido explicaciones bastante heterogéneas sobre la violencia (Wieviorka, 2001), algunas la han reflexionado desde lo estructural, centrando su atención en los procesos de diferenciación y luchas sociales, la coerción y las desigualdades, sin poner atención en la acción violenta. También se ha revisado desde el orden normativo, en donde la violencia es la representación simbólica del poder (Arteaga y Arzuaga, 2017). Para otras la violencia es el resultado de las crisis de un sistema, en tanto que para el enfoque racional la violencia es el resultado de una acción instrumental de los actores, del cálculo de un actor individual y por tanto estratégico (Wieviorka, 2001; Fisas, 1987), esto le imprime un alto de grado de subjetividad al victimario, pero también a la víctima (Álvarez, 2013). La vertiente culturalista encuentra la explicación de la violencia en el peso de la cultura como generadora de la personalidad individual y colectiva de una sociedad (Chenais, 1981; Arteaga, 2013).

Al respecto cabe hacer alusión a lo que señala Dos Santos (2020), en el sentido de que los "fenómenos culturales generan una violencia cultural y social que se combina con la formación ideológica liberal de fondo que justifica y estimula la competencia individualista como base ética del sujeto social" (Dos Santos, 2020, p. 271).

Esto permite visualizar la existencia de distintas interpretaciones, sin embargo, para la sociología el problema se ubica en la articulación entre los procesos estructurales que condicionan la violencia y el sujeto que genera la acción violenta. Esto implica que la influencia de los elementos estructurales, como las variables económicas y políticas, se tienden a sobrestimar y se subestima el cálculo racional del actor que ejerce la violencia, que valora esta acción en términos de costo-beneficio (Arteaga, 2013).

Para Wieviorka (en Arteaga, 2013), la interpretación estructuralista establece que la violencia es la consecuencia de alguna forma de crisis social, que produce frustraciones 
individuales y colectivas, debido al funcionamiento, disfuncionalidad, o transformación del sistema. El enfoque utilitarista en cambio analiza la violencia como un medio que utiliza el individuo para allegarse de bienes y servicios que no obtendría por otra vía, la violencia desde esta perspectiva es una acción instrumental orientada por una acción racional de costobeneficio ${ }^{5}$, este enfoque es el que desarrolla Arendt (2006). La visión culturalista de la violencia, como ya se dijo, le asigna a la cultura un papel fundamental en la construcción de la personalidad individual y colectiva de una sociedad. A través de estos enfoques se pueden observar ciertos aspectos de la violencia, pero esconden otros.

En tal sentido, de acuerdo con Wieviorka (en Arteaga, 2013), estas tres perspectivas ubican al actor como un individuo que responde a las condicionantes en las que está inmerso. Por tanto, Wieviorka (2001), ubica al sujeto en el centro del análisis, enfatizando en la heterogeneidad de la violencia, así como en sus significaciones y las distintas formas en que se relaciona con el sujeto, al ser constitutiva de él, esto conlleva subjetivización y desubjetivización de los actores que ejercen la violencia. Por tanto, la violencia no es más que la marca del sujeto contrariado, negado o imposible, la marca de una persona que ha sufrido una agresión, sea física o simbólica...entonces la violencia, en algunos casos, no es más que la incapacidad del sujeto de convertirse en actor 6 (Wieviorka, 2001, p. 340).

La vertiente antropológica del estudio de la violencia se puede observar por medio de dos enfoques fundamentalmente: a) el primero, vinculado con el estudio de las culturas de la violencia, los cuales se enfocan en los ritos, costumbres, subculturas y organizaciones que se estructuran con base en determinados códigos para el uso legítimo o ilegítimo de la violencia, ya sea interpersonal 0 autoinfligida; b) el segundo, centra su atención en el análisis de las violencias de la cultura, que se identifica a través de las violencias que se producen en instituciones o campos culturales como la política, la vida cotidiana, los aspectos estructurales o microsociales, así como las violencias física, simbólica, visible, invisible, experimentada e imaginada. El primer enfoque es el tradicional en esta materia, el segundo, analiza la violencia desde la perspectiva de la microfísica del poder (Ferrándiz y Feixa, 2004).

Para la antropología política (Balandier, 1979), lo social es resultado del manejo de la violencia, por medio de ritos, normas y símbolos, la cual es regulada a través de diversas instituciones, entre los que destacan los dispositivos jurídico-legales que establecen una relación de obediencia entre la voluntad y poder del Estado y la de los gobernados (Foucault, 2006).

Según Galtung (2003), la violencia es como un iceberg, solo es visible una pequeña parte; por tanto su solución implica actuar en los tres tipos de violencia que la caracterizan: la violencia directa, la violencia estructural y la violencia cultural.

Desde la psicología, una conducta violenta puede manifestarse de dos formas: 1) Violencia expresiva. Es el tipo de conducta agresiva expresada a través de la ira, dirigida a una víctima conocida y que denota ciertos problemas en el control o expresión de las emociones (celos, envidia, odio, etc.); por tanto, después de ese acto se presenta alguna forma de

\footnotetext{
${ }^{5}$ Este enfoque se encuentra en los trabajos teóricos de Wieviorka, Tilly, Joas y Collins (Arteaga, 2013).

${ }^{6}$ Para Wieviorka (2001) la violencia es constitutiva del sujeto, pues a la vez que construye sentido también se da una pérdida de sentido con ella; es desubjetivización, pero también subjetivización. Pero también reconoce la existencia de una violencia ligada a la crisis del sistema, a la crisis del Estado. Esta crisis la liga a la incapacidad del Estado para impedir las agresiones o los robos por ejemplo, porque si las instituciones estatales no son capaces de detenerlo se experimentará un incremento.
} 
arrepentimiento, y 2) Violencia instrumental. En este caso la conducta agresiva esta guiada por una lógica de costo-beneficio, por consiguiente está encaminada a conseguir un objetivo específico, la víctima suele ser desconocida y no hay culpa por el daño ocasionado (Echeburúa y Amor, 2010). La explicación del estado de violencia que se genera por las pasiones artificiales se manifiesta en la presencia o ausencia de una conducta cooperativa entre los hombres (Dieterlen, 1998).

Entre los autores de la psicología social, Klineberg (1981), propone una lectura crítica sobre las diferentes aproximaciones de la violencia para distinguirla de la agresividad, debido a que la agresividad humana es definida por lo regular como el uso de la fuerza contra otra persona o sus bienes con el propósito de causar daño, definición que también se utiliza para el vocablo violencia.

Desde la Teoría Política, la violencia es fundadora del Estado porque conserva para sí el control y ejercicio de la violencia (Weber, 1991), en este sentido para Weber (1991), el Estado es la

asociación de dominación con carácter institucional que ha tratado, con éxito, de monopolizar dentro de un territorio el monopolio ${ }^{7}$ de la violencia legítima como medio de dominación y que, con este fin, ha reunido todos los medios materiales en manos de sus dirigentes y ha expropiado a todos los seres humanos que antes disponían de ellos por derecho propio, sustituyéndolos con sus propias jerarquías supremas (Weber, 1991, p. 92)

En este sentido, de acuerdo con Weber el objetivo del Estado es preservar su estabilidad y su supervivencia y para cumplir con dicho objetivo el uso de la violencia es fundamental. Arendt (2006) se aleja de esta postura porque para ella "la violencia puede ser justificable pero nunca será legítima. Su justificación pierde plausibilidad cuanto más se aleja en el futuro del fin propuesto (Arendt, 2006, pp.71-72).

Cercano al planteamiento de Weber, para Castells (2009), el poder es la capacidad relacional que permite a un actor social influir de forma asimétrica en las decisiones de otros actores sociales, de modo que se favorezcan la voluntad, los intereses y los valores del actor que tiene el poder ${ }^{8}$. Por lo tanto, el poder es un recurso que se ejerce mediante la coacción -0 la posibilidad de ejercerla- y mediante la construcción de significado, partiendo de los discursos a través de los cuales los actores sociales guían sus acciones. "Para el Estado la capacidad relacional que ofrece el poder está determinada por las instituciones, por medios coactivos y disciplinarios, esto significa la institucionalización de la violencia" (Nateras y Martínez, 2018, p. 242). No obstante, con ello se genera una relación de unilateralidad y perjuicio (Garriga y Noel, 2010).

De acuerdo con Castells (2009), la institucionalización de la violencia es posible a través de las instituciones y de la disciplina, de esta forma el Estado establece las reglas y normas para mantener su estabilidad interna y ejercer su poder hacia afuera. Para Foucault (1999 y

\footnotetext{
${ }^{7}$ De acuerdo con Mbembe (2011) el monopolio de la violencia ha dejado de ser exclusivo del Estado, en la actualidad la gestión de la violencia y el derecho a matar puede ser utilizado por ejércitos privados para poder competir con el poder del Estado.

8 "Para entender la emergencia de la violencia a partir de la interacción social Tilly introduce los conceptos de mecanismos y procesos de la violencia...los mecanismos son 'causas en escalas pequeñas' que hacen posible una mejor apreciación de los detonantes de la violencia y que desplazan las interpretaciones de la violencia vinculadas a 'grandes causas' como la pobreza, la cultura y las tradiciones - también a factores como la frustración y la competencia por recursos. Por otro lado, los procesos son 'combinaciones y secuencias de mecanismos que producen efectos similares a través de amplios espectros de circunstancias' (en Arteaga, 2013, p.p. 41-42).
} 
2002) la disciplina funciona como dispositivo de vigilancia y control. No obstante, "las sociedades no son comunidades que compartan valores e intereses. Son estructuras contradictorias surgidas de conflictos y negociaciones entre diversos actores sociales, a menudo opuestos" (Castells, 2009, p. 38). Al respecto, "es importante destacar que el poder es relacional y la dominación es institucional" (Nateras y Martínez, 2018, p. 243).

Considerando los planteamientos anteriores, el ejercicio del poder político siempre es violento, no importa cómo y a través de qué medios lo ejerza. Al respecto, Esposito (2006), señala que los conceptos políticos desarrollados por Hobbes ${ }^{9}$, representan una forma gramatical, propia del liberalismo, para denominar filosófica y políticamente el argumento biopolítico que justifica la protección de la vida de los individuos, debido a que están expuestos a una amenaza constante y únicamente de esta forma se puede evitar su ocaso de manera violenta. En este sentido, en la biopolítica se condensa el poder y la vida, es decir, a través de ella se delinean los esfuerzos encaminados a regir la vida en general de los individuos por parte del Estado. Son las instituciones del poder como la familia, el ejército, la escuela, la policía, la medicina, entre otras, las que aseguran y garantizan la permanencia de las relaciones de dominación y por tanto la hegemonía (Foucault, 1997). El ejercicio del poder tiene sus particularidades que se pueden observar a través de las técnicas disciplinarias de control y coacción sobre el conjunto de la población (Foucault, 1997 y 2007).

La violencia, a diferencia del poder o la fuerza, siempre necesita herramientas porque está tutelada por la categoría medios-fines y debido a su naturaleza instrumental requiere de una justificación para su uso en aras del objetivo que persigue. La violencia no puede derivarse del poder, en realidad son opuestos, y más aún la violencia puede siempre destruir al poder (Arendt, 2006). Para Benjamin (2001), en cambio, si la violencia fuera solo un medio para satisfacer un fin seria totalmente infructuoso su uso para instituir o transformar contextos de manera estable.

El poder, en tanto relacional, es la facultad y el medio para ejercer influencia sobre otros de forma directa, y casi siempre coercitiva, por lo tanto el resultado es la conformación de relaciones asimétricas entre los individuos. El objetivo del poder es establecer una posición hegemónica para tener el dominio y con ello construir la legitimidad necesaria que garantice su permanencia, para ello, se vale de las leyes y los dispositivos de vigilancia que los gobiernos diseñan para proteger la vida de los individuos, pero sobre todo para garantizar su permanencia, y así lo institucionaliza. En esta lógica discursiva, el biopoder se visibiliza a través de los mecanismos disciplinarios que se han construido para vigilar y dominar los cuerpos humanos, los cuales están sostenidos por todo un entramado jurídico (Esposito, 2006; Foucault, 2002).

Respecto al último punto, retomando a Agamben (2004 y 2006), los controles jurídicoconstitucionales deben estar orientados a crear o definir el espacio mismo en que el orden jurídico-político ya institucionalizado reúne derecho y violencia, es decir el nomos soberano, que también tiene potestad para legitimar la excepción, es decir, el espacio libre y jurídicamente vacío (Nateras y Martínez, 2018, p. 250).

\footnotetext{
${ }^{9}$ De acuerdo con la tesis de Hobbes, en el "estado de naturaleza" todos los hombres tienen el suficiente poder para causar daño al otro; por tanto, aunque vaya en contra a su naturaleza de buscar la libertad y la de ejercer dominio sobre los demás, al final terminan autorestringiéndose y optan por el establecimiento de la sociedad, la ley y Estado, por una razón utilitaria. El objetivo final es salir de la lamentable y temida condición de guerra, y optar por la autoconservación es una decisión racional (Zavatta, 2016)
} 
De acuerdo con el primer planteamiento de Agamben (2006), para Benjamin (2001), la violencia es fundadora de derecho, esa es su primera función, pero viéndola como un medio tiene una doble función: ser fundadora de derecho y al mismo tiempo utilizar la violencia para conservar el derecho. Desde este planteamiento, "fundación de derecho equivale a fundación de poder, y es, por ende, un acto de manifestación inmediata de la violencia. Justicia es el principio de toda fundación divina de fines; poder, es el principio de toda fundación mítica de derecho" (Benjamin, 2001, p. 40). Toda violencia fundadora de derecho garantiza el poder.

"Este es el poder soberano que concentra la violencia, elabora las leyes, las aplica y está facultado para ser el juez supremo" (Ávalos, 2020, p. 212). A través del poder soberano, se mantiene el control de la sociedad (Hardt y Negri, 2000).

\section{La violencia como objeto de estudio: la propuesta de Žižek}

Desde la filosofía política, se retoman los planteamientos de Slavoj Žižek, específicamente en su obra "Sobre la violencia. Seis reflexiones marginales", en cuyo texto Žižek (2009), esboza las diferentes formas de la violencia en las sociedades actuales. Centrando su atención en la manera en que el sistema capitalista media las relaciones sin que sea posible percatarse de ello, haciendo que la violencia esté presente en todos y cada uno de los ámbitos de la vida (violencia omnipresente). Algunos aspectos centrales se complementan con su libro "En defensa de la intolerancia" (2008).

Hoy en día la violencia, en todas sus formas de expresión y manifestación, se ha vuelto tan cotidiana que cada vez es más difícil de distinguir por su grado de invisibilización y porque ésta suele estar asociada a todas las formas en que se manifiesta la violencia directa ${ }^{10}$. Sin embargo, el problema es más complejo; por ello Žižek (2009), considera que es necesario que nos detengamos a reflexionar en cómo las condiciones que se entienden normales están determinadas por otras formas de violencia, como las violencias sistémica y simbólica. El problema es que el proceso de comprensión que las sociedades llevan a cabo frente a una expresión de violencia concreta termina por invisibilizar las causas que le dan origen.

En este sentido, para Žižek (2009), la violencia que él denomina objetiva es invisible porque sostiene la normalidad y a partir de ella se construye la forma hegemónica de concebir la violencia y es la que legitimamos. La naturalización o normalización de la violencia se apoya en la violencia simbólica ${ }^{11}$, la cual está incorporada en el lenguaje y sus formas ${ }^{12}$, y en la violencia sistémica ${ }^{13}$ que se genera en los sistemas económico y político, y que, además, está presente en las formas más sutiles de coerción que imponen las relaciones de dominación y explotación, así como en las relaciones sociales que impone el sistema capitalista, lo que hace imposible que no nos percatemos de ella, por eso es que señala que la violencia objetiva está anclada a la génesis del capitalismo, para mantener el orden de las cosas.

\footnotetext{
${ }^{10} \mathrm{El}$ riesgo de designar como violentos solo a las acciones físicas en una confrontación es lo que termina por invisibilizar aquellas formas sutiles de victimización y coerción (Noel, 2008).

${ }^{11}$ A la violencia simbólica se le relaciona normalmente con el concepto marxiano de dominación, por tanto por medio de ella los sujetos dominados aceptan y reproducen su sometimiento (Taussig, 1987).

12 Para Morrison "El lenguaje opresivo hace algo más que representar la violencia; es violencia, produce su propio tipo de violencia" (en Butler,1997: 21).

13 Žižek $(2008,2009)$ entiende la violencia sistémica a partir de dos conceptos: la biopolítica y la pospolítica. La biopolítica es la regulación de la seguridad y el bienestar de las vidas humanas, que se centra en defender del acoso potencial del "otro"; por ello es, en última instancia, una política del miedo. La pospolítica se concentra en la administración de una multitud paranoide y atemorizada por la presencia de "el otro".
} 
Diferenciar las violencias subjetiva y objetiva es fundamental, pues mientras para Wieviorka (2001) la violencia objetiva es la real y la violencia subjetiva es la que se percibe, desde la postura de Žižek $(2008,2009)$, la violencia subjetiva es aquella que podemos ver, la que se manifiesta diariamente a través de los actos violentos y crueles ${ }^{14}$ y que es ejercida por diversos actores, es la que rompe con el desarrollo "normal" de las cosas, para Benjamin (2008) esta es la violencia pura o divina. Los protagonistas de esta violencia sobre los individuos pueden ser los aparatos del Estado, individuos o colectivos sociales. Para Žižek (2009), entre más hable de violencia la sociedad, se cuestiona cada vez menos acerca de ella. Es por ello que la violencia subjetiva, que apunta de modo directo a los actos de violencias concretas que se vive cotidianamente a través de los medios de comunicación masiva 0 directamente, es la violencia.

En este sentido, a través de la violencia simbólica (Žižek, 2008 y 2009), se impone el discurso del grupo hegemónico y la ideología del propio sistema, de esta forma se vuelve etéreo y se acallan al otro y sus voces, para normalizar ${ }^{15}$ la violencia sistémica ${ }^{16}$; es decir, por medio de la violencia simbólica se hace evidente la violencia subjetiva y la distancia de la violencia sistémica. La violencia sistémica se puede observar de manera clara cuando los ricos y poderosos gozan de privilegios e impunidad; cuando ante esos beneficios actúan en detrimento de sus trabajadores, de la naturaleza, y en general de la humanidad, actuando solo en su beneficio. Esta forma de violencia no es imputable a individuos concretos, es anónima, objetiva, sistémica, por lo tanto, es más extraña a cualquier otra forma de ejercicio de la violencia directa.

El problema es que la violencia simbólica se intenta eliminar a través de la violencia sistémica, aún cuando ésta la estimula. En consecuencia, si no se combate la violencia sistémica y con ello se apunta a rescindir las violencias subjetivas, se logrará que se extiendan las tres: sistémica, simbólica y subjetiva. Esto hace que nos percatemos y nos sorprendamos cuando se incrementa el número de delitos, como indicador tangible de la violencia.

No obstante, como advierte Žižek (2009), los actos violentos y las víctimas de actos atroces no tienen el mismo valor para nosotros, unos actos son más condenables que otros, sesgando así el análisis frente al problema de la violencia, por ello lo urgente y lo importante para los hombres está mediado por lo político. No es lo mismo la tortura, una ejecución o el ejercicio de la violencia física aplicado a una persona o un grupo de personas consideradas transgresoras del orden hegemónico establecido, que aquellas personas "respetuosas" del status quo, sobre todo cuando se trata defender la seguridad mundial.

En términos políticos y económicos, de acuerdo con Žižek (2009), estamos inmersos en un sistema que nos afecta y violenta, pero a la vez se muestra como el generador de las soluciones a los problemas ocasionados por él mismo. Por lo tanto, el Estado, quien detenta el poder sobre el ejercicio de la violencia, también la genera e imparte, es el responsable de velar

\footnotetext{
${ }^{14}$ Reflexionando sobre la crueldad, como algo inexplicable e irracional, Wieviorka (2001) retoma a Primo Levy, quien como judío y que conoció las experiencias del campo de concentración, se preguntaba en sus textos por qué los guardianes de los campos nazis eran crueles y su conclusión era la siguiente: "desde el punto de vista del nazi que estaba en el campo, era necesario que antes de morir la víctima fuera degradada...'Para poder lograr lo que voy hacer, yo tengo que suprimir aquello que hace que el otro sea sujeto... es necesario que yo lo trate como una bestia; que yo lo envilezca; que yo lo destruya. Para atarme al sentimiento de ser sujeto, es necesario que al otro no lo trate como tal, como a un ser humano'. Por eso la crueldad hace a la persona que es cruel, un anti-sujeto" (p.p. 344-345).

${ }^{15}$ Ejemplo de esta naturalización son: racismo, homofobia, discriminación, misoginia, xenofobia, etc.

${ }^{16}$ Ejemplos de esta violencia son: miseria, desigualdad, exclusión, delincuencia, etc.
} 
por el bienestar de los ciudadanos, en calidad de víctimas directas de esta violencia, esto nos coloca frente a una paradoja. Un claro ejemplo en el terreno económico es la manera en como el sistema capitalista perpetúa la violencia económica a través de ejercicios discursivos, invitado a consumir con el argumento del consumo como requisito para el mejoramiento de la calidad de vida de los más necesitados. Por consiguiente, somos víctimas de un sistema reconocido como benefactor y no como victimario, en tal sentido el autor habla de la legitimidad e ilegitimidad de la violencia.

En esa misma idea, Žižek (2008, 2009), plantea que el capitalismo ha construido el discurso de la tolerancia liberal y éste es la causa última de la violencia, el cual se refleja a través del miedo al prójimo: miedo a sentir al otro como invasor de mi espacio, como una amenaza, esto se traduce en miedo al inmigrante, al negro, o a cualquier otro diferente, que se visualiza en la actitud intimidante. Por tanto, el otro deja de ser sujeto para convertirse en un objeto a quien se puede dañar de manera justificada, siempre que ello evite una cuota de dolor mucho mayor para el resto de la población, bajo el argumento del bien común. En este sentido la tolerancia es una categoría ideológica y en el centro de ésta se encuentra el lenguaje del respeto, pero como lenguaje propio de la tolerancia liberal, el respeto sólo tiene significado como respeto para aquellos con los cuales no estoy de acuerdo.

La actual tolerancia liberal hacia los demás, el respeto a la alteridad y la apertura hacia ella, se complementa con un miedo obsesivo al acoso. Dicho de otro modo, el «otro» está bien, pero sólo mientras su presencia no sea invasiva, mientras ese otro no sea realmente «otro» (Žižek, 2009, p. 57).

Cuando las afectaciones son colectivas, se vuelve más aceptable los actos violento contra quienes están propiciando esa afectación, pues es preferible atacar el mal, no importa si es por medio del uso de la violencia, de esta manera resulta menos ominoso. Esto, porque como sociedad, somos sensibles a diferentes manifestaciones de violencia, al mismo tiempo que somos tolerantes e inclementes a ciertas formas de violencia orientada a solidarizarnos con las víctimas. En estos casos, señala Žižek (2009), preferimos cargar con el peso de este tipo de acciones violentas y hacer como si no nos diéramos cuenta que estamos legitimando el uso de la violencia. Porque ante situaciones de desorden y violencia, el sentido de amenaza es activado, surgiendo así una necesidad latente de estigmatizar al otro, de convertirlo en enemigo.

El discurso hegemónico, como generador de la violencia simbólica es el encargado de sostener la existencia del enemigo, el cual en el contexto de la globalización, se convierte en el enemigo universal y es en este punto en el que las fronteras se abren o se cierran, para acoger o rechazar al otro y en caso de ser abiertas, los primeros opositores serían quienes ya se han ganado un lugar al pasarlas ${ }^{17}$. Žižek (2009), afirma que el reto, en este sentido, es lograr un cambio en la sociedad, para poder transformar el sistema socioeconómico y no sea éste el responsable de violentar y expulsar a los ciudadanos hacia otros lugares, es decir, para impedir un huida desesperada de su propio mundo.

\footnotetext{
${ }^{17}$ Un ejemplo claro de esto es el discurso racista y antiinmigrante de Trump, que le permitió ganar el apoyo de los latinos que migraron hace años a ese país, temerosos que ante la llegada al poder de la candidata demócrata y la posibilidad de la apertura de las fronteras para la acogida de los migrantes perdieran todos los beneficios y privilegios logrados hasta ahora.
} 
Las contradicciones del discurso de la tolerancia las encontramos también en los actos violentos que se producen cuando se estigmatiza a un credo religioso, o a un país, aún cuando éste haga gala de tener un alto nivel de tolerancia, el cual resistiría la prueba de la provocación; cuando esta provocación se produce se trastocan de manera violenta los parámetros básicos de la vida social, generando una respuesta violenta. No obstante, el resultado esta vinculado con los actos y sus contextos, es decir, es producto de la relación entre la violencia subjetiva y sistémica, debido al contexto como factor fundamental en la percepción de la violencia.

Si con el término "violencia" queremos designar el trastorno radical de las relaciones sociales básicas, entonces, por muy disparatado o de mal gusto, el problema de los monstruos históricos que asesinaron a millones de personas es que no fueron los suficientemente violentos. A veces no hacer nada es lo más violento que puede hacerse (Žižek, 2009, p. 256).

Para entender esto Žižek (2009), recurre a ejemplificar con todas las personas que han muerto debido al sistema capitalista, entre éstas las víctimas del holocausto nazi, en esos actos de horror siempre existirán distintas interpretaciones, entre ellas la del fundamentalismo radical ${ }^{18}$, esta postura sostiene que "el holocausto no ocurrió; los que señalan que ocurrió pero los judíos se lo merecían; los judíos no se lo merecían, pero perdieron su derecho a lamentarse cuando hicieron a los palestinos lo que los judíos les hicieron a ellos" (Žižek, 2009, p. 136). Todas estas afirmaciones, desde cualquier perspectiva, son reprobables, pues la única referencia posible del holocausto, es la negativa; no para argumentar la importancia de estas estrategias, sino para desaprobar algunas medidas o restringir ciertas decisiones políticas.

Sobre todo cuando se piensa en una violencia con aires de divinidad, para analizar ésta Žižek (2009), introduce o retoma el concepto de violencia divina desarrollado ampliamente por Benjamin $(2001,2008)$, quien la concibe como una forma emancipatoria de la violencia, cuya característica principal es orientar a la sociedad hacia el futuro y aliviarla de sus males. No obstante, para Žižek (2009), la violencia divina no es una explicación religiosa, o cercana al dogma religioso, es más bien la aceptación de que un cambio intempestivo va más allá de un trastorno infructuoso, para poder alinear las cosas con rumbo a la libertad, como en máximo valor del respeto por el otro.

La violencia divina emana de la injusticia, la impotencia y de la violencia sistémica, por ello debe desligarse de su sentido mítico, este tipo de violencia se identifica con los fenómenos históricos existentes. Se trata de un "signo sin significado, y la tentación que debemos resistir es...la tentación de proporcionarle un sentido profundo" (Žižek, 2009, p.p. 236-237).

La violencia mítica es un medio para establecer el dominio de la ley (el orden social legal), mientras que la violencia divina no sirve a ningún medio, ni siquiera al castigo de los culpables para así restablecer el equilibrio de la justicia (Žižek, 2009, p. 236).

\footnotetext{
18 Žižek (2009) determina que a los "pseudofundamentalistas", simplemente los mueve el resentimiento de quienes se han surtido de ser "inferiores" a los occidentales, quienes se acogen a la cultura occidental pero se odian asimismo por no ser occidentales, por ese motivo, confirma el autor su tesis primaria de que no cuentan con otra fuente ideológica y por ello recurren a la violencia.
} 
La violencia mítica está inscrita al espacio del ser y la violencia divina en cambio es parte del acontecimiento, no obstante no hay elementos para definir o reconocer un hecho derivado de la violencia divina (Žižek, 2009).

Según Benjamin (2001), el origen primigenio de la violencia mítica deviene de la manifestación de la voluntad y de la existencia de los dioses, por ende es fundadora de derecho; asimismo, establece fronteras, es culpabilizadora, expiatoria, amenazadora, es sangrienta en su propio nombre y debido a esto exige sacrificios. En tanto, la violencia divina es destructora de derecho, derriba las fronteras, pero se muestra como redentora, pero cuando golpea es letal, aunque se muestre apacible acepta sacrificios aún cuando profesa amor a lo vivo. Dicha violencia divina se manifiesta en la expresión sacralizada de la vida cotidiana, una de sus manifestaciones más común es la violencia educadora.

Žižek $(2008,2009)$, orienta sus críticas a la ilusión de que en el liberalismo somos libres de tomar nuestras propias decisiones y a la falsa universalidad encubridora de los privilegios de unos cuantos. Un ejemplo es la quimera de la universalidad de los derechos humanos, los cuales bajo el manto de los derechos para todos legitima y encubre la lógica de la dominación. En el fondo, para Žižek esto es una muestra de la simulación del poder que usa y abusa de su supremacía a través de la coacción y la apropiación de los recursos, recordando con ello a los oprimidos que son ellos la causa de sus propios males y no al abuso de sus opresores.

\section{Conclusiones}

Para entender la violencia este texto se propuso hacer una revisión de cómo es abordada por distintas disciplinas, así como por distintos autores, en especial Žižek. Pero como se pudo ver, su abordaje no es sencillo, desde una postura restringida, la violencia se asocia al uso de la fuerza física, consecuentemente es un acto interpersonal, con la intención de provocar daño físico. Asociadas a la Teoría Política, están las posiciones legitimistas que conciben a la violencia como inherente al Estado y aceptan el uso de la coacción física. Por otro lado, existen definiciones más amplias que consideran a la violencia como parte de la estructura socio-cultural y por consiguiente asociadas a situaciones de dominación. No obstante, para esta última vertiente, violencia y conflicto es lo mismo, debido a la naturaleza violenta del conflicto.

Sin embargo, como se pudo leer en este artículo, erradamente se piensa que la violencia desintegra o divide los intereses de los individuos, sin embargo, es el conflicto quien ha hecho previamente esta labor, debido a que la violencia mana cuando no se cuenta con otros instrumentos para resolver el conflicto; esto implica tener claro a la violencia como resultado del conflicto y no al revés, porque un conflicto puede existir sin que jamás se desplieguen actos de violencia.

No obstante, desde la Sociología, la principal crítica a las distintas interpretaciones de la violencia es la falta de articulación entre los procesos estructurales condicionantes de la violencia y el sujeto generador de la acción violenta. Este planteamiento permitió establecer la diferencia entre las violencias objetiva y subjetiva, para Žižek la violencia objetiva es la real, no obstante es invisible, porque sostiene la normalidad y al estar legitimada, a partir de ella se construye la forma hegemónica de concebir la violencia. Asimismo, para el mismo autor, la violencia subjetiva es aquella que se manifiesta cotidianamente a través de los actos violentos 
concretos y crueles ejercida por los actores sociales y por los aparatos del Estado sobre la ciudadanía, en consecuencia es visible.

Pero qué es realmente la violencia objetiva, siguiendo con la propuesta de Žižek, la violencia objetiva se naturaliza o normaliza por el apoyo de las violencias simbólica y sistémica. La primera está incorporada en el lenguaje y la segunda se produce en los sistemas económico y político, a través de los sistemas de dominación y explotación, pero también en las relaciones sociales impuestas por el sistema capitalista. La violencia simbólica permite la imposición del discurso e ideología de la clase dominante, esto invisibiliza y silencia las voces del otro, al mismo tiempo que naturaliza la violencia sistémica. La violencia simbólica permite visibilizar la violencia subjetiva y la desconecta de la violencia sistémica, por tanto, las violencias física y simbólica están enlazadas.

Un ejemplo es la forma en como el sistema capitalista, a través del discurso, alienta al consumo con el argumento de su contribución a mejorar la calidad de vida de los más necesitados, sin considerar los factores que impiden este consumo y termina generando frustración, violentando a grandes capas de la sociedad imposibilitadas de contar con los medios para consumir. Otro ejemplo, es la forma en cómo se justifica el dolor o la violencia contra algunas personas, bajo el argumento del bien común, es decir, cuando se arguye que la intención es evitar una cuota de dolor mayor para el resto de la población. Cuando las afectaciones son colectivas, se vuelve más aceptable la violencia contra quien o quienes están generando el mal. Un caso concreto es el homicidio del general iraní Qasem Soleimani el 3 de enero de 2020 , orquestado por EEUU, so pretexto de que estaba planeando un ataque a ese país.

Este discurso hegemónico construye la idea de un enemigo común, el cual se convierte en el enemigo universal en el contexto de la globalización y es quien cierra 0 abre fronteras, a través de éste se construye la idea del peligro de un migrante por ejemplo. Asimismo, señala al terrorismo y al narcotráfico como los principales enemigos a nivel mundial. En este sentido, para Žižek, la hipocresía de los Imperios se observa básicamente a través de dos actitudes: porque utilizan su supremacía para hacer uso de su poder coactivo y para legitimar la expropiación de los recursos de otros territorios, y por otro lado, les restriegan a los oprimidos ser el resultado de su propia barbarie innata y sus problemas sociales son consecuencia de eso no del abuso de los dominadores.

El problema que oculta la violencia simbólica es su pretensión de combatirla haciendo uso de la violencia sistémica provocada por ella. En consecuencia, si no se combate la violencia sistémica y con ello se apunta a rescindir las violencias subjetivas, se logrará que se extiendan las tres: sistémica, simbólica y subjetiva.

\section{Referencias Bibliográficas}

Agamben, Giorgio. (2004). El estado de excepción. Pre-textos. España.

Agamben, Giorgio. (2006). Homo Sacer: El poder soberano y la nuda vida. Pre-textos. España.

Álvarez, Santiago. (2013). ¿A qué llamamos violencia en las ciencias sociales? Hallazgos, 10(20), Colombia (Pp. 61-71). DOI: https://doi.org/10.15332/s17943841.2013 .0020 .05

Arendt, Hannah. (2006). Sobre la violencia. Alianza Editorial. España. 
Aróstegui, Julio. (1994). Violencia, sociedad y política: la definición de la violencia. Ayer, Asociación de Historia Contemporánea and Marcial Pons Ediciones de Historia, Vol. 13, España (Pp. 17-55). Extraído de https://www.researchgate.net/publication/254133288_Violencia_sociedad_y_politica_I a definicion de la violencia

Arteaga, Nelson; Arzuaga, Javier. (2017) Sociologías de la violencia. Estructura, sujetos, interacciones y acción simbólica. Flacso, México.

Arteaga, Nelson. (2013).

Fermentum, Revista Venezolana de Sociología y Antropología, 23 (66), Venezuela (Pp. 33-56). Extraído de http://www.saber.ula.ve/handle/123456789/39886 Ávalos, Gerardo. (2020). El monarca, ssepel ciudadano y el excluido. UAM, México.

Balandier, George. (1979). Violence et anthropologie. En Maffesoli, Michel; Bruston, André (dir.). Violence et transgression. Anthropos. Francia.

Benjamin, Walter. (2001). Para una crítica de la violencia y otros ensayos (tercera edición). Taurus. España.

Benjamin, Walter. (2008). "Hacia la crítica de la violencia". En Tiedemann, Rolf; Schweppenhäuser, Hermann (Eds.), Obras, II, 1, (Pp. 183-206). Abada. España.

Butler, Judith. (1997). Lenguaje, poder e identidad. Ed. Síntesis. España.

Castells, Manuel. (2009). Comunicación y poder. Alianza. España.

Chenais, Jean-Claude. (1981). Histoire de la violence. Robert Laffond (ed.). Francia.

Coady, Cecil. (1986). The Idea of Violence. Journal of Applied Philosophy, 3(1), U.S.A. (Pp. 3-19).

Coser, Lewis. (1961). Las funciones del conflicto social. F.C.E. México.

Dahrendorf, Ralf. (1962). Las clases sociales y su conflicto en la sociedad industrial. Ediciones Rialp. España.

De Sousa, Boaventura. (2003). Crítica de la razón indolente: Contra el desperdicio de la experiencia. Editorial Desclée de Brouwer, S.A. España.

Dieterlen, Paulette. (1998). Hobbes: el porqué y para qué de la violencia. Facultad de Filosofía y Letras. UNAM, Fondo de Cultura Económica. México.

Domenach, Jean-Marie. (1981). La violencia. En La violencia y sus causas. UNESCO. Francia.

Dos Santos, Theotônio. (2020). SEEP Construir soberanía : una interpretación económica de y para América Latina. CLACSO. Argentina.

Echeburúa, Enrique; Amor, Pedro J. (2010). Perfil psicopatológico e intervención terapéutica con los agresores contra la pareja. Revista Española de Medicina Legal, 36(3), España (Pp.117-121). https://doi.org/10.1016/S0377-4732(10)70040-7

Esposito, Roberto. (2006). Bios, biopolítica y filosofía. Amorrortu. Argentina.

Fanon, Frantz. (2007). Los Condenados de la Tierra. FCE. México.

Ferrándiz, Francisco; Feixa, Carles (2004). Una mirada antropológica sobre las violencias. Alteridades. 14(27), México (Pp. 159-174). Extraído de https://www.redalyc.org/pdf/747/74702710.pdf

Fisas, Vicenç. (1987). Introducción al estudio de la paz y de los conflictos. Lerna. España. Foucault, Michel. (1997). Historia de la sexualidad I: La voluntad de saber. Siglo XXI Editores. México. 
Foucault, Michel. (1999). Estrategias de poder. Paidós. Argentina.

Foucault, Michel. (2002). Vigilar y castigar. Nacimiento de la prisión. Siglo XXI Editores. Argentina.

Foucault, Michel. (2006). Seguridad, territorio, población. Curso en el Collège de France: 1977-1978. FCE. Argentina.

Foucault, Michel. (2007). La gubernamentalidad. En Giorgi, Gabriel; Rodríguez, Fermín. Ensayos sobre biopolítica: excesos de vida. Paidós. Argentina.

Galtung, Johan. (2003). Tras la violencia, 3R: reconstrucción, reconciliación, resolución. Afrontando los efectos visibles e invisibles de la guerra y la violencia. Gernika: Bakeaz/Gernika Gogoratuz. España.

Garriga, José; Noel, Gabriel. (2010). Notas para una definición antropológica de la violencia: un debate en curso. Publicado, en Antropología y Ciencias Sociales, 8(9), Argentina (Pp. 121-126). Extraído de https://www.academia.edu/2219747/Notas_para_una_Definición_Antropológica_de_I a Violencia_Un Debate en Curso

Hardt, Michael; Negri, Antonio. (2000). Imperio. Harvard University Press, Cambridge. USA. Harris, Olivia. (1994). Condor and Bull. The ambiguities of masculinity in Northern Potosí. En Harvey Penelope; Gow, Peter. Sex and violence. Issues in representation and experience. Routledge. USA.

Harvey, Penelope. (1994). Domestic violence in the Peruvian Andes. En Harvey, Penelope; Gow, Peter. Sex and violence. Issues in representation and experience. Routledge. USA.

Incháustegui, Teresa; López, María de la Paz. (2012). Violencia feminicida en México.

Características, tendencias y nuevas expresiones en las entidades federativas, 1985-2010. ONU Mujeres, INMujeres y LVI legislatura, Cámara de Diputados. México.

Isla, Alejandro; Míguez, Daniel. (2003). Heridas Urbanas. Violencia Delictiva y

Transformaciones Sociales en los Noventa. Editorial de las Ciencias. Argentina.

Klineberg, Otto. (1981). Las causas de la violencia desde un perspectiva socio-psicológica. En

La violencia y sus causas. UNESCO. Francia.

Mbembe, Achille. (2011). Necropolítica. Melusina. España.

Moore, Henrietta. (1994). The problem of explaining violence. En Harvey, Penelope; Gow, Peter. Sex and violence. Issues in representation and experience. Routledge. USA.

Nateras, Martha; Martínez, Oscar. (2018). La seguridad nacional en México: entre legitimidad y violencia de Estado. Veredas. Riesgo y seguridad, reflexiones desde la Sociología, 36 (19), México (Pp.239-262). Extraído de http://www.casadelibrosabiertos.uam.mx/contenido/contenido/Libroelectronico/vereda s-36.pdf

Noel, Gabriel. (2008). Versiones de la violencia. Las representaciones nativas de la violencia y su reconstrucción analítica en las escuelas de barrios populares urbanos. Propuesta Educativa, (30), Argentina (Pp. 101-108 Extraído de https://www.redalyc.org/pdf/4030/403041702010.pdf 
Osorio, Sergio. (2012). "Conflicto, violencia y paz: un acercamiento científico, filosófico y bioético". Revista Latinoamericana de Bioética. 12(2), Colombia (Pp. 52-69) Extraído de https://www.redalyc.org/pdf/1270/127025833006.pdf

Pérez, Germarilin. (2017). Manifestaciones y Factores de la Violencia en el Escenario Escolar. Telos: revista de Estudios Interdisciplinarios en Ciencias Sociales, 19(2), Venezuela (Pp. 237-259). Extraído de http://ojs.urbe.edu/index.php/telos/article/view/150/135

Pessin, Alain. (1979). Violence et transgression. Éditions anthropos. France.

Platt, Thomas. (1992). La violencia como concepto descriptivo y polémico. En Revista Internacional de las Ciencias Sociales. Pensar la Violencia: perspectivas filosóficas, históricas, psicológicas y sociológicas. España. (Pp. 173-180). Extraído de https://unesdoc.unesco.org/ark:/48223/pf0000091531_spa

Rex, John. (1985). El Conflicto Social. Siglo XXI editores. España.

Riches, David. (1988). El fenómeno de la violencia. Pirámide. España.

Sagot, Montserrat. (2013). El femicidio como necropolítica en Centroamérica. LABRYS, Études Féministes/Estudos Feministas. Brasil. Extraído de https://www.labrys.net.br/labrys24/feminicide/monserat.htm

Sorel, Georges. (2005). Reflexiones sobre la violencia. Alianza Editorial. España.

Taussig, Michael (1987). Shamanism, Colonialism, and the Wild man. A study in terror and healing. University of Chicago Press. USA.

Weber, Max. (1991). El político y el científico. Alianza editorial. España.

Wieviorka, Michel. (2001). La violencia: Destrucción y constitución del sujeto. Espacio Abierto, 10 (39), Venezuela (Pp. 337-347). Extraído de https://www.redalyc.org/pdf/122/12210301.pdf

Zavatta, Laura. (2016). La visione moderna del diritto e dello Stato da Hobbes e Nietzsche alla sfida della mondialisation. Telos: revista de Estudios Interdisciplinarios en Ciencias Sociales, 18(2), Venezuela (Pp. 318-341). Extraído de http://ojs.urbe.edu/index.php/telos/article/view/758/694

Žižek, Slavoj. (2008). En defensa de la intolerancia. Ediciones sequitur. España.

Žižek, Slavoj. (2009). Sobre la violencia. Seis reflexiones marginales. Paidós. España. 